\title{
Left transverse venous sinus thrombosis associated to pneumococcal meningitis in children - intuitive vs. cognitive diagnosis
}

\author{
Maria Obreja' ${ }^{1}$ Liliana Vlad' ${ }^{1}$, Radu Miftode ${ }^{2}$, Alexandr Ceasovschih ${ }^{3}$, Egidia Miftode ${ }^{1,2}$ \\ 1"Sfanta Parascheva" Infectious Diseases Hospital lasi, Romania \\ 2"Grigore T. Popa" University of Medicine and Pharmacy, lasi, Romania \\ ${ }^{3}$ Department of Internal Medicine, "Sf. Spiridon" Emergency Clinical Hospital, Iasi, Romania
}

\begin{abstract}
Introduction. Cerebral venous thrombosis (CVT) is a rare cerebrovascular condition involving multiple etiologies. Diagnosis of CVT is usually overlooked or delayed due to highly varied symptomatology, modes of onset and neuroimaging signs; furthermore, the same cause cannot be found in more than $15 \%$ of the cases, individual result being hard to predict, while the disease can complicate despite anticoagulant treatment. Material and methods. We present a typical case of meningitis that in day 10 of illness associated an uncommon symptomatology suggesting cerebral venous sinus thrombosis (CVST).

Results. It should be noted that imaging examination can neither confirm nor exclude a thrombosis. A case whose development worsens progressively and for which there is no imaging exam to support a particular therapeutic conduct hampers the decision-making process for the physician.

Discussion. The issue raised focuses on the assumption that anticoagulant medication may be beneficial to the patient or, on the contrary, it may be in the detriment of the physician who is uncertain about the diagnosis, due to subsequent unfavorable development of patient clinical status.

Conclusions. That human rational thinking, exercised in years of experience, cannot yet be technologized, let alone replaced by a robot, and the existence of sets of rules for treatment incapable to ever cover the variety of features encountered in practice must always be doubted.
\end{abstract}

Keywords: cerebral vein thrombosis, meningitis, seizures, computer-tomography, diagnosis

\section{INTRODUCTION}

Cerebral venous thrombosis (CVT) is a cerebrovascular condition affecting 5 persons to $1,000,000$ and represents $0.5 \%$ of total cerebral vascular accidents (1). First recognized in the early $19^{\text {th }}$ century, CVT has an incidence comparable to that of acute bacterial meningitis in adults (2), but it is less common than ischemic vascular accident or intracerebral hemorrhage. It usually affects the superior sagittal sinus, causing unilateral or bilateral focal neurologic deficits, seizures and coma, which, most of the times, lead to death.

In the last 25 years, high-speed neuroimaging contributed to early diagnosis of pathology, which amended the current information that exists about this disease. CVT is recognized as a non-infectious disease with varied clinical manifestations and favourable response, with mortality well below $10 \%$. Magnetic resonance and angiography are the best methods of diagnosis and heparin is used as firstline treatment (1).

\section{CASE PRESENTATION}

We hereby describe the case of a 10 -year-old boy, from rural areas, presented in the Pediatric Emergency Department with fever, productive cough, frontal headache, vision changes, vertigo, vomiting and abdominal pain. The disease had an 
insidious onset during the 5 days preceding hospitalization, eventually with lack of response to stimuli associated with loss of consciousness. From the personal medical history, we must mention recurrent respiratory tract infections, overactive bladder syndrome, iron deficiency anemia and stature-ponderal hypotrophy.

A computer-tomography (CT) exam was conducted in the Department of Neurology of the Pediatric Hospital where a diffuse cerebral edema was revealed. Biological examinations showed inflammatory syndrome associated with leukocytosis and neutrophilia $(\mathrm{GA}=33,490 / \mathrm{mmc}$ and $\mathrm{PMN}=93.1 \%$, FBG: $467 \mathrm{mg} / \mathrm{dl})$. Clinical context in conjunction with laboratory data and CT scan non-indicative of a neurosurgical condition raised the suspicion of meningitis, therefore the patient was directed to the Infectious Diseases Hospital with suspected meningitis.

Upon admission, patient's clinical status was severely marked, with fever, Glasgow coma score (GCS) 4-5, intense meningeal contraction syndrome: positive Kernig's sign I and Brudzinski's neck and contralateral reflex signs, facial congestion, tachycardia, urinary incontinence. A lumbar puncture was performed, providing the following data on the cerebrospinal fluid (CSF): cloudy, with 960 cells $/ \mathrm{mm}^{3}$ of which $\mathrm{PMN}=97 \%$, with the presence of $S$. pneumoniae.

Treatment with vancomycin and cefotaxim was initiated, but the antibiogram subsequently confirmed sensitivity for these antibiotics. Due to the emergence of an erythematous eruption on face, neck and thorax as a result of rapid administration of vancomycin, the therapeutic scheme was modified, replacing vancomycin with rifampicin (according to antibiogram). On the third day of hospitalization a new lumbar puncture revealed a cloudy CSF with 3,400 cells $/ \mathrm{mm}^{3}$ of which $\mathrm{PMN}=98 \%$. Antibiotic therapy with cefotaxim was interrupted and meropenem was introduced.

After two days, the patient was affected by a right-sided hemiparesis with uncoordinated movements of the right upper limb, absence of deep tendon reflexes, and positive Babinski sign. The suspicion of a brain abscess prompted for a new CT exam. The diagnosis was "observation of left transverse sinus thrombosis near the origin and mild diffuse cerebral edema", without neurosurgical indi- cation. Despite diagnostic uncertainty following the CT examination, anticoagulation treatment with enoxaparinum was initiated.

Anticoagulant treatment with enoxaparinum was administered. On day 6 from admission another lumbar puncture showed a favourable trend of inflammatory reaction from CSF with the presence of 1,270 cells $/ \mathrm{mm}^{3}$ of which PMN $=88 \%$. Due to the persistence of seizures at the level of the right hemibody, which have not succumbed to diazepam, and the psychomotor agitation later associated, followed by the lack of cooperation and altered mental status (GCS 4-5), the patient was admitted to the intensive care unit for surveillance, for 4 days. During this time the motor deficit was improved and the coma evolved towards a superficial stage. In this time the antibiotic treatment was the same. He was re-admitted to the ward, where he presented visual hallucinations, but they resolved spontaneously.

After 12 days, treatment with meropenem was stopped and cefotaxime was reintroduced. A new lumbar puncture was performed on day 18 from admission revealing clear CSF with 10 cells $/ \mathrm{mm}^{3}$ and complete blood count within the normal values: $\mathrm{WBC}=7,380 / \mathrm{mm}^{3}, \mathrm{PMN}=60.4 \% \mathrm{PCR} \leq 6 \mathrm{mg} / \mathrm{l}$, $\mathrm{ESR}=40 \mathrm{~mm} / \mathrm{h}$. A new CT exam was carried out, without revealing any changes.

The patient was discharged after 33 days of hospitalization, clinical status improved, with good general condition, spatially and temporally oriented, cooperative, without skin rash, with intermittent pain in the right lower limb, force and mobility preserved.

\section{DISCUSSIONS}

The present case was approached as a case of the CVST, and the initiation of anticoagulant therapy associated with antibiotic and symptomatic treatment resulted in a favorable trend.

Considered as the meningitis-associated CVST, the case presented sustains the data in the literature regarding the dynamics required to diagnose such a case. The rapid evolution, with focal neurologic phenomena, stresses the need for monitoring by repeating the imaging examinations even though the CSF exam appears to indicate towards a refractory purulent meningitis, with the increase of leukocytes 
and neutrophilia in patients with unfavorable evolution.

Therefore, it is known that CVT is more frequent in young people, mostly children, than any other type of stroke. Among patients of childhood age, there is an increased incidence of CVT among new born babies (3), predominantly of the female sex (2), a fact which is not sustained by our case.

CVT may occur in the superficial or deep venous system and venous sinuses. The superficial venous system is more frequently involved (75\%-85\%) compared to the deep venous system (14\%-38\%). There are anatomical differences that make the superficial venous system more susceptible to thrombosis. Underdiagnosed thrombosis in the deep venous system involvement may be accounted for by the limits of radiological examination.

CVT is associated with multiple acute and chronic conditions. These include fever, infections, dehydration, anemia, as well as acute or chronic disorders such as systemic lupus erythematosus, renal disease, leukemia, blood conditions and congenital heart defects (4). However, according to Carpenter et al., between 23 and $65 \%$ of children were reported as being in good health prior to diagnosis (5).

Thrombophilia, genetic or acquired, is an important risk factor. Despite recommendations for thrombophilia-screening, in all children with CVT independent of other risk factors, only a few cases have been reported as having a prothrombotic factors assessment. The number of patients with CVT and an identified risk factor for thrombophilia vary from study to study, between $33 \%$ and $99 \%$. This percentage should be interpreted with precaution because the studies are too small to have statistical power (5).

Infectious causes of thrombosis are diminishing but in developing countries, however, systemic infections and infections of the central nervous system remain an important cause of cerebral vein thrombosis. The most common infections associated with thrombosis are otitis, mastoiditis, and sinusitis [2]. Meningitis can be complicated by the CVT, which increases diagnostic difficulty, reason for which incidence remains underestimated (4). Pneumococcal meningitis is associated with intracranial complications which occur in about $74.7 \%$ of adult patients, $9.2 \%$ of whom are represented by the CVT (6).

Heller et al. (7) reported trauma as a risk factor in $6.7 \%$ of the children with CVT. Studies have shown that thrombosis associated to trauma occurs more frequently in the transverse or superior sagittal sinus but without a correlation with the severity of injury (5).

As risk factor among those mentioned in the literature, our patient has a history of anemia, but has not been investigated for thrombophilia. The likelihood of a thrombotic process can be accounted for by the context of infective meningitis. Anamnesis data also have not reported any cranial injury.

Clinical symptomatology tends to vary depending on age and associated factors. New-borns and young children most often have seizures and lethargy. Older children present in particular symptoms of increasing intracranial pressure such as headache, vomiting and altered mental status. Headache is the most common symptom, usually the first, it could be the only manifestation. They can also present focal neurological signs (hemiparesis, cranial nerves palsy) and seizures (5). Coma can be seen as a symptom at presentation in $28 \%$ of children with CVT (3).

The patient's initial symptoms were interpreted in the context of meningitis. As soon as the motor deficit was observed in day 10 of illness, a new CT was recommended. CT examination result rose the suspicion of left transverse sinus thrombosis with persistence of diffuse cerebral edema.

Motor deficit, altered mental status and seizures may plead in favour of complications by CVST, as inflammatory reaction from CSF examinations was diminishing, but the clinical condition of the patient worsened visibly as described.

Clinical diagnosis of CVT is difficult to establish and is based on non-invasive methods such as CT, CT venogram, MRI, MR venogram, conventional angiogram or transcranial Doppler. Conventional angiogram is considered the gold standard, however, the $\mathrm{CT}$ exam remains the most frequently used $(5,8)$. In our case, the diagnostic imaging exam was the CT.

A logical question arising from the unlikelihood of imaging diagnosis is why there was no additional investigation with superior diagnosis sensitivity for CVST, from those just mentioned? The answer 
can only be one: reduced access to additional and expensive diagnostic methods, in the context of a developing country economy. For this reason, physician's clinical intuition weighs considerably on the border between pathologies, in the evaluation of a clinical case.

Specific therapy for CVT involves anticoagulation and supportive therapy. Adequate treatment of infection, dehydration, fever, increased intracranial pressure, seizures, blood pressure abnormalities, hyper or hypoglycaemia can have a significant impact (4).

Basically, our approach to the case as a meningitis-associated CVST required as a therapeutic measure the initiation of anticoagulant medication considering that for this approach there was no clear imaging support. The issue raised by this case focuses on the assumption that anticoagulant medication may be beneficial to the patient or, on the contrary, it may be in the detriment of the physician who is uncertain about the diagnosis, due to subsequent unfavorable development of patient clinical status.

Anticoagulation therapy can be an effective in CVT and is well tolerated by children, although there are no studies to prove its effectiveness. Anticoagulant therapy is successful in rechannelling the occluded vein, limiting the spread of the existing clot as well as the formation of other thrombi, thus preventing pulmonary embolism. One must gain this advantage in order to limit the risk of hemorrhage $(7,9)$.
Anticoagulation effectiveness was evaluated in only two small randomized trials. It was shown some benefit of anticoagulants in intracerebral vein thrombosis and, perhaps most importantly, the safety of anticoagulant therapy (10).

Our case has received antibiotherapy covering the spectrum of antimicrobial-resistant $S$. pneumoniae to penicillin, but also a staphylococcus perhaps, a micro-organism with the most associations with thrombotic events (8). He has also been treated with enoxaparinum. Therapeutic measures, on the whole, resulted in a slow development, with improvement of the motor deficit and coma status.

\section{CONCLUSIONS}

A relatively rare complication described in the literature, CVST associated with Streptococcus pneumoniae meningitis must not influence the therapeutic decision based on clinical intuition, all the more so that it is reported as an underdiagnosed pathology. The evolution of the case was slow, with symptoms remission, thus demonstrating that human rational thinking, exercised in years of experience, cannot yet be technologized, let alone replaced by a robot, and the existence of sets of rules for treatment incapable to ever cover the variety of features encountered in practice must always be doubted.

\section{Conflict of interest: none declared} Financial support: none declared

\section{REFERENCES}

1. Bousser M.G., Ferro J.M. Cerebral venous thrombosis: un update. Lancet Neurol. 2007; 6:162-70.

2. Ferro J.M., Canhão P. Cerebral venous sinus thrombosis: update on Diagnostic and management. Curr Cardiol Rep. 2014; 16:523.

3. Sébire G., Tabarki B., Saunders D.E. et al. Cerebral venous sinus thrombosis in children: risk factors, presentation, diagnosis and outcome. Brain. 2005; 128:477-89.

4. Dlamini N., Billinghurst L., Kirkham F.J. Cerebral venous sinus (sinovenous) thrombosis in children. Neurosurg Clin N Am. 2010; 21:511-527.

5. Carpenter J., Tsuchida T. Cerebral sinovenous thrombosis in children. Curr Neurol Neurosci Rep. 2007; 7:139-146.

6. Kastenbauer S., Pfister H.W. Pneumococcal meningitis in adults:spectrum of complications and prognostic factors in a series of 87 cases. Brain. 2003; 126:1015-1025.

7. Ferro J.M., Canhão P. Acute treatment of cerebral venous and dural sinus thrombosis. Curr Treat Options Neurol. 2008; 10:126-137.

8. Sasidharan P.K. Cerebral Vein Thrombosis Misdiagnosed and Mismanaged. Thrombosis. 2012; Article ID 210676.

9. Lindgren A. Long-term prognosis of cerebral vein and sinus thrombosis. Front Neurol Neurosci. 2008; 23:172-178.

10. Einhäupl K., Bousser M.G., de Bruijn S.F., Ferro J.M., Martinelli I., Masuhr F., Stam J. EFNS guideline on the treatment of cerebral venous and sinus thrombosis. Eur J Neurol. 2006; 13(6):553-9. 